\title{
Radiological manifestations from 101 patients with 2019 Novel Coronavirus Disease (COVID-19) in Hamadan, West of Iran: Typical and atypical findings
}

\section{Farideh Gharekhanloo}

Hamadan University of Medical Sciences Medical School

\section{Seyyed Hamid Hashemi}

Hamadan University of Medical Sciences Medical School

\section{Fariba Keramat}

Hamadan University of Medical Sciences Medical School

Farzaneh Esna-Ashari

Hamadan University of Medical Sciences Medical School

Mojgan Mamani

Hamadan University of Medical Sciences Medical School

Mohammad Mahdi Majzoubi

Hamadan University of Medical Sciences Medical School

\section{Peyman Eini}

Hamadan University of Medical Sciences Medical School

\section{Mahsa Gharekhanloo}

Yazd University

\section{Salman Khazaei ( $\sim$ salman.khazaei61@gmail.com )}

Hamadan University of Medical Sciences School of Public Health

\section{Research article}

Keywords: Chest CT, COVID -19, Coronavirus, chest radiograph, Iran

Posted Date: April 25th, 2020

DOI: https://doi.org/10.21203/rs.3.rs-19678/v1

License: (c) (i) This work is licensed under a Creative Commons Attribution 4.0 International License.

Read Full License 


\section{Abstract \\ Background}

Coronavirus disease 2019 (COVID-19) has spread widely throughout the world and become a pandemic disease. In this study we decided to investigate the chest computed tomography (CT) findings in COVID19 patients in Hamadan, west of Iran.

\section{Methods}

This cross-sectional study was conducted on 101 patients with confirmed COVID-19 infection from February to March 2020. Demographic, clinical, laboratory and chest CT information of identified COVID19 patients were assessed.

\section{Results}

Totally, 101 patients diagnosed with COVID-19 were included. The mean age of the patients was $55.21 \pm$ 14.08 years and $54 \%(53.47 \%)$ of them were male. With regards to clinical manifestations, $82.18 \%$, $72.28 \%$ and $54.46 \%$ of COVID-19 patients had dry cough, dyspnea and fever, respectively and $44.5 \%$ had lymphopenia. The right lower lobe was the most common (69\%) and severe involved lobe followed by left lower lobe, right middle lobe, and lingual; however, anterior segment of upper lobes showed least involvement with abnormality in the late course of disease. The most common pattern is ground glass opacity, but atypical patterns such as round pneumonia, moderate to severe plural effusion and segmental lobar collapse consolidation was seen without evidence of mediastinal adenopathy, cavitation or nodule. Chest X ray (CXR) was not sensitive method as a first line imaging because $34.65 \%$ of them were normal.

\section{Conclusion}

CXR is not sensitive method as first line imaging too (34.65\% normal first CXR), but Chest CT is very sensitive and nonspecific modality for diagnosis of COVID - 19. Lower lobe and posterior basal predominance involvements were seen in most cases. About $12 \%$ showed atypical chest CT features.

\section{Background:}

An outbreak of coronavirus disease 2019 (COVID-19) infection was first expanded in December 2019 in Wuhan, the capital of Hubei province of China and is being exported to a growing number of countries (1). Alongside severe acute respiratory syndrome (SARS) and Middle East respiratory syndrome (MERS), COVID-19 is another common type of coronavirus that infect humans (2). The transmission way of 
COVID-19 is direct contact from human to human, mainly through respiratory droplets, and also through contact by the same mechanism as other common cold or influenza viruses (3).

The symptoms of COVID-19 infection appear after an incubation period, that studies so far suggest a mean incubation period of five days (range: 0-24 days) (4). The most common presenting clinical symptoms of COVID-19 are fever, dry cough, dyspnea, muscle soreness, and fatigue $(5,6)$. Some of the patients reported have headache or hemoptysis and some have relatively asymptomatic $(7,8)$.

Approximately $80 \%$ of COVID-19 infections are mild or asymptomatic, $15 \%$ are severe infection requiring oxygen and $5 \%$ are critical infections requiring ventilation and mortality is approximately $3 \%(9)$. The older patients are more likely to have respiratory failure due to severe alveolar damage.(10). The old age, male sex, and presence of comorbidities are high risk factors for poor prognosis of the disease (1).

Real time- polymerase chain reaction (RT-PCR) technique has high false negative rate and needs at least 48 hours for determination, while chest CT has high sensitivity and gives the results in the small fraction of the time $(11,12)$. Therefore, chest CT plays an important role in early detection of chest abnormality in suspected case. These features of image CT help physicians for rapid management, planning, initiation of treatment, isolation of patients and determine severity of disease. Previous studies showed that imaging plays an important role in follow up of COVID-19 patients (13). There are few studies on imaging of COVID-19 patients and majority of them belong to China, therefore we conducted this study to observe the imaging characteristics of the COVID-19 patients in Iran.

\section{Methods:}

This cross-sectional study was performed in March 2020 in Hamadan Province, the west of Iran. The number of 101 patients with confirmed COVID-19 infection identified in all hospitals in the province affiliated to Hamadan University of Medical Sciences were enrolled in the study. Hamadan province with an area of 19493 square kilometers in extent is located in west of Iran and had a population of 1,758,268 people; according to the national census held in 2016 by Statistical Center of Iran:

http://irandataportal.syr.edu/census/census-2016

The Ethics Committee of Hamadan University of Medical Sciences was approved the study. Patients with positive RT-PCR were considered as inclusion criteria and were assessed. It should be noted that these patients have not been reported in any other submission by us or anyone else.

A checklist contains demographic, clinical, laboratory findings and chest CT examination of identified COVID-19 patients were used for collecting data. Demographic and clinical data including signs, symptoms and underlying diseases history, were asked directly from the patients and when the patient was unable to respond due to a bad physical condition, the information was taken with the physician or referring to patient medical records. Biochemical blood test results were gathered by refer to the patient's medical record. 
For each patient chest images were reviewed independently. All hospitalized patient underwent chest computed tomography (CT Scan) using a multi-detector CT scanner with Somatom Emotion 16 slice channels (Siemens Healthcare, Erlangen, Germany) obtained in supine position and deep inspiration state without injection of contrast media. Acquisition parameters was as following characteristics: $170-200$ MAs; $110-120 \mathrm{kVp}$; collimation $0.625-5 \mathrm{~mm}$ and pitch, 1.2. Images were reconstructed with slice thickness $0.625-5 \mathrm{~mm}$ and reconstruction interval $1-3 \mathrm{~mm}$ sharp reconstruction kernel all images viewed with lung (width, $1500 \mathrm{HU}$; level, $-600 \mathrm{HU}$ ) and mediastinal window (width, $400 \mathrm{HU}$; level, $40 \mathrm{HU}$ ).

Two expert radiologists (with 11 and 20 years of experience in chest imaging, respectively) analyzed and reviewed all the chest radiographs and CT images on a picture archiving and communication system (PACS Marco) by consensus on controversial images. All images were evaluated for this parameters: presence of lesion, location and pattern of lung abnormality (ground glass opacity, patchy mix consolidation reticular interlobular septal thickening segment and lobe involvement, mostly and minimally involved lobe presence or absence of plural and pericardial effusion, lymphadenopathy cavitation, underlying lung disorder, presence or absence of reverse halo sign and crazy paving appearance. Ground glass opacity is defined as increased lung density without obscuration of vascular and bronchial margin due to partial filling of air space (14). Consolidation is increased density with obscuration of bronchial and vascular structure (14). Reverse halo sign of at all sign is described as partial ring or rim consolidation around the focal ground glass opacity. Crazy pacing appearance is defined as interlobular septal thickening in the background of ground glass opacity. Peripheral is defined as one third of periphery of lung in trans axial plane (14).

For data analysis we used descriptive statistics including: mean (SD) for describe the continuous variables and counts and percentage for describe the categorical variables arranged in frequency table and chart. Data were analyzed using Stata 14 (StataCorp, College Station, TX, USA).

\section{Results:}

The total number of 101 COVID-19 patients with the mean age of $55.21 \pm 14.08$ years (range: $27-$ 87 years) were investigated. Fifty-four (53.47\%) were male. The age distribution of the patients is shown in Fig. 1.

Less than half had underlying diseases (36 patients [35.64\%]): including cardiac disorder, diabetes, chronic obstructive pulmonary disease, asthma and hypertension in $15.84 \%, 8.91 \%, 3.96 \%$ and $2.97 \%$ of cases, respectively (Fig. 2).

The main clinical symptoms at the early stage of illness were dry cough in 83 cases (82.18\%), dyspnea in 73 cases $(72.28 \%)$ and fever in 55 cases $(54.46 \%)$. In regards of laboratory results, 45 (44.5\%) had lymphopenia and in vast majority of them CRP were 2 plus. Clinical and laboratory findings of investigated patients are presented in Table 1. 
Table 1

Clinical and laboratory findings in the COVID-19 patients

\begin{tabular}{|lll|}
\hline Variables Characteristics & N (\%) \\
\hline Signs and symptoms & Fever & $55(54.46)$ \\
\cline { 2 - 3 } & Dry cough & $83(82.18)$ \\
\cline { 2 - 3 } & Sore throat & $4(3.96)$ \\
\cline { 2 - 3 } & Dyspnea & $73(72.28)$ \\
\hline Laboratory & Lymphopenia & $45(44.5)$ \\
\hline Thrombocytopenia & $8(7.92)$ \\
\hline CRP & Anemia & $17(16.83)$ \\
& Negative & $18(17.82)$ \\
\cline { 2 - 2 } & + & $61(60.40)$ \\
\hline++ & $17(16.83)$ \\
\hline+++ & $5(4.95)$ \\
\hline
\end{tabular}

Among total cases, common CT features were ground glass opacity (GGO), inter lobular septal thickening and patchy consolidation in $64.35 \%, 33.66 \%$ and $26.73 \%$ cases, respectively. Other CT features is shown in Fig. 3.

Findings at initial chest CT examination in the COVID-19 patients are presented in Table 2. Of the 101 patients, 99 patients $(98.02 \%)$ had bilateral involvement, but two of them had unilateral. Ninety-six patients $(95.05 \%)$ had asymmetrical laterality and the right lobe was involved in $68.32 \%$ of them. At the first chest CT of the patients, involvements were seen in the right upper, middle and lower lobes, 39 (32.67\%), 13(12.67\%) and 69 (68.32\%), respectively. However, the left upper and lower lobes were involved in $33(38.61 \%)$ and $58(57.43 \%)$, respectively. The most and least involvements were seen in the right lower lobe and right middle lobe in $58(57.43 \%)$ and 30 (29.70\%) of cases, respectively. In regards of CXR, 35 (34.65\%) patients had normal CXR at the first day of diagnosis. In relation to other radiographic features of the patients it can be noted that $10(9.9 \%)$ cases had mild plural thickening, 2 patients $(1.98 \%)$ had pneumothorax, but 6 of them (5.94\%) had mild to moderate plural effusion and $2(1.98 \%)$ patients with severe plural effusion. 
Table 2

Imaging characteristics at the initial chest CT in the COVID-19 patients

\begin{tabular}{|c|c|c|}
\hline \multicolumn{2}{|l|}{ Variables Characteristics } & \multirow{2}{*}{$\begin{array}{l}\mathbf{N}(\%) \\
2(1.98)\end{array}$} \\
\hline Lung involvement & Unilateral & \\
\hline & Bilateral & $99(98.02)$ \\
\hline \multirow[t]{2}{*}{ Laterality } & Symmetrical & $5(4.95)$ \\
\hline & Asymmetrical & $96(95.05)$ \\
\hline \multirow[t]{3}{*}{ Involved lung } & Both lobe & $5(4.95)$ \\
\hline & Right lobe & $69(68.32)$ \\
\hline & Left lobe & $27(26.73)$ \\
\hline \multirow[t]{7}{*}{ Lung lobes involvement } & Left Lower Lobe & $58(57.43)$ \\
\hline & Left Upper Lobe & $33(38.61)$ \\
\hline & Right Upper Lobe & $39(32.67)$ \\
\hline & Right Middle Lobe & $13(12.87)$ \\
\hline & Right Lower Lobe & $69(68.32)$ \\
\hline & Lingual & $12(11.88)$ \\
\hline & All & $52(51.49)$ \\
\hline \multirow[t]{7}{*}{ The most involvement } & None & $1(0.99)$ \\
\hline & Left Lower Lobe & $29(28.71)$ \\
\hline & Left Upper Lobe & $6(5.94)$ \\
\hline & Right Upper Lobe & $9(8.91)$ \\
\hline & Right Middle Lobe & $6(5.94)$ \\
\hline & Right Lower Lobe & $58(57.43)$ \\
\hline & Lingual & $3(2.97)$ \\
\hline \multirow[t]{5}{*}{ The least involvement } & None & $6(5.94)$ \\
\hline & Left Lower Lobe & $9(8.91)$ \\
\hline & Left Upper Lobe & $12(11.88)$ \\
\hline & Right Upper Lobe & $18(17.82)$ \\
\hline & Right Middle Lobe & $30(29.70)$ \\
\hline
\end{tabular}




\begin{tabular}{|lll|}
\hline Variables Characteristics & $\mathbf{N}(\%)$ \\
\hline & Right Lower Lobe & $3(2.97)$ \\
\cline { 2 - 3 } & Lingual & $28(27.72)$ \\
\hline
\end{tabular}

\section{Discussion:}

COVID-19 is a novel coronavirus disease caused by SARACov2 that spread in the world. In the present study, 101 patients were enrolled. The average age of the patients was 55.21 years with ranging from 27-87 years. Thirty-six percent had underlying or coexisting medical disorders including diabetes, cardiac disease, hypertension, COPD, asthma and end stage renal disease. This is different from influenza pneumonia which involved older patients with further underlying disorders $(15,16)$.

The most common symptom was dry cough (83\%) followed by dyspnea (73\%). Only $55 \%$ of the patients had fever and lymphopenia, anemia and thrombocytopenia were seen in $45 \%, 17 \%$ and $8 \%$ respectively. Moreover, chest CT scan had only $3.9 \%$ misdiagnosis of COVID - 19 and should be considered as a rapid diagnosis tool.

In the study of Shi et al. showed the presence of ground glass opacities in 15 asymptomatic patients and suggested CT scan is a sensitive modality even in patient with false negative RT- PCR results and highly suspicious clinical findings (17). So according to high false negative rate of RT- PCR $(18,19)$ and high sensitivity of CT scan (up to 98\%) (20) chest CT scan is accepted as a first screening tool for diagnosis of Covid-19 pneumonia. Another imaging modality is chest $\mathrm{x}$ ray however; chest $\mathrm{x}$ ray is not as sensitive as chest CT in evaluation of lung abnormality as first line of diagnostic tool in suspected cases. In the present study about $35 \%$ of the patients showed normal CXR as first imaging modality and $65 \%$ showed some nonspecific abnormal findings. According to Soon Ho Yoon et al. study, the most of Korean COVID19 patients had normal chest $x$ ray or ambiguous findings (21) and in Chinese COVID-19 patients chest radiographic abnormalities were seen in only in $60 \%$ of patients. Soon $\mathrm{Ho}$ Yoon et al.(21) also emphasized that findings of imaging is milder than two other similar viral infection (SARS and MERS-Cov pneumonia), and $33 \%$ of the COVID-19 cases had abnormal initial CXR while in the SARS and MERS-Cov infection $78.3-82.4 \%$ and $83.6 \%$ of initial CXR were abnormal, respectively (21). So, all of clinician should be familiar to limitation of CXR as a first line imaging tool in evaluation of COVID - 19 pneumonia.

Another study on 121 chest CT by Adam Bernhiem et al. $56 \%$ of the patients in the early stage (0-2 days after symptom onset) had normal chest CT scan (2). In this study, chest CT scan that was performed in 2-4 days after symptom onset, lung abnormality was seen in the entire chest CT scan (100\% of cases). CT findings of the patients were generally consistent with those of COVID-19 pneumonia patients in China (13).

In the present study, two patients had unilateral right lung disease (only in right lower lobe) and $98.02 \%$ showed bilateral lung involvement and in $95.05 \%$ of them asymmetrical involvement was seen. The 
predominant lung involvement was right lung (69\%), but in $27 \%$ of the patients, left lung involvement was dominant and $5 \%$ of them showed symmetrical abnormality in both lungs. In the study of Bernheim et al. reported that $20(17 \%)$ patients had unilateral lung involvement, but left and right lungs involvement were separately seen in 7 and 13 patients, respectively (2).

The hallmarks of novel coronavirus infection on imaging are bilateral GGO mostly peripheral, followed by mixed patchy multifocal opacity and/or consolidation. However, in the study of Adam Bernheim et al. $22 \%$ of patients had no ground -glass opacity and no consolidation (2), while in our study $55 \%$ of patient showed ground-glass opacity and $36 \%$ showed mixed ground glass opacity and patchy consolidations. In the pictorial review study by Zheng Ye et al. pure ground glass opacity between 14-96\% and mixed ground glass opacity and consolidation between $19-59 \%$ were reported (22).

In this study predominant abnormality was seen in right lower lobe, which is the first involvement. Posterior, peripheral and subpleural abnormalities were dominant features and anteriorly located segments and lobes such as right middle lobe, an lingula and anterior segment of upper and lower lobes showed less frequent, and less severe abnormality and often this lobes and segments abnormality was seen in severe cases and later in the course of disease. In the another systematic review of imaging findings of COVID-19 pneumonia, GGO is seen mainly in the lower lobe and less frequently in the middle lobe (23). However, in the present study, right lower lobe abnormality is often early and the first radiologic finding in COVID-19 pneumonia. In the study of Wu J et al. (24) the most frequent pattern was GGO (91\%) followed by consolidation (63\%) and interlobular septal thickening (59\%).

In fact, this peripheral GGO with or without mixed patchy consolidation is not characteristics for COVID19 pneumonia. Patchy ground glass opacity and consolidation with a predominantly subpleural and/or peribronchial distribution and lower lobe predominance are the most common imaging features in organizing pneumonia in CXR and chest CT scan however can affect all lung zone $(25,26)$, so the involvement of lungs in COVID-19 pneumonia is categorized as organizing pneumonia.

Most pulmonary lesion in the viral infection was seen with peripheral and posterior lung predominant distribution and this is not a characteristic finding for COVID-19 pneumonia. Distribution of SARS and $\mathrm{H} 1 \mathrm{~N} 1$ is also peripherally located $(27,28)$. In the another study of Yan Li et al. on 53 patients, 51 patient confirmed case of COVID-19 (with Nucleic acid testing) and two proved case of adenovirus CT findings were similar to COVID-19 cases and showed ill-defined patchy GGO with segmental and subpleural distribution (29). Yan Li et al. (29) were noted the ground glass pattern and coalescence in to consolidation with predominance of lung periphery are similar to SASR and MERs infection and some other studies emphasizes the non-specificity of chest CT finding.

According to study of Koo $\mathrm{HJ}$ et al. influenza pneumonia affected the lung with lower lobe predominance (30). Wang et al. also emphasized that prominent and dominant distribution of H7N9 influenza pneumonia is the right lower lobe (15). This is nonspecific pattern of organizing pneumonia as immune reaction and corticosteroids might suppress and disappear this type of lung abnormality. However, there is difference between influenza and COVID-19 pneumonia in comparison with influenza pneumonia 
complete consolidation in COVID-19 pneumonia is less common. In the study by Wang Q et al. most patients with H7N9 pneumonia showed complete consolidation (15).

Another point about the pattern of lung involvement is that chest CT scan manifestation is altered with the course of disease and severity (18-19). In the present study, in mild cases and in the early stage of disease, GGO was predominant pattern and later with the progression of disease, the pattern followed by crazy paving and consolidation in chest CT. In addition, Shi et al.'s study (31) showed progression of GGO to bilateral diffuse consolidation at first to second weeks of infection after symptom onset and peripheral and posterior lung involvements were seen $78 \%$ and $67 \%$ of cases, respectively. In addition, the most of lesions were ill defined, composed of consolidation and ground glass opacities, and lower lobe predominance was also seen and less common nodular pattern was long the broncho vascular bundles. In the study on 121 chest CT by Adam Bernhiem et al. (2) there was a correlation between onset of clinical symptoms and chest CT scan and bilateral typical peripheral involvements in 0-2days: early, 3-5 days: intermediate and 3-12 days: late was observed in 28,78 and $88 \%$ of patients, respectively.

Reticular thickening as interlobular septal thickening is seen due to interstitial lymphocyte infiltration (18). This is the second frequent lesion in COVID-19 pneumonia $(24,31)$. In the present study, $33 \%$ of patients showed interlobular septal thickening. Another atypical and less common finding was plural changes that as plural thickening and plural effusion were seen. The former is more frequent, $32 \%$ versus $5 \%(31)$.

In this study, plural thickening was seen in $10 \%$ and mild to moderate plural effusion was seen in $6 \%$. Two cases in the end stage of disease showed moderate to severe plural effusion. Reverse halo sign is seen in $5 \%$ of the patients. In the study of Bernheim et al. and Yoon et al. reported reverse halo sign in 2 and $3 \%$ of COVID-19 patients $(2,21)$. This sign first described as specific finding in cryptogenic organizing pneumonia; however, recently believed this sign might have indicated an organizing pneumonitis due to some viral or fungal infection such as COVID-19 pneumonia (32).

In this study, other less frequent and atypical findings were seen as follow: $5 \%$ of the cases showed crazy paving appearance mostly in severe cases, $5 \%$ of patients had only one lobe of segment involvement, with segmental or lobar collapse consolidation appearance, one case with peribroncho vascular infiltration, one case with typical round pneumonia, mid generalized ground glass opacity in the entire lung was seen in one patient.

There was no case with significant lymph adenopathy, cavitation or nodular lesion or broch ectasia. Air bronchogram only was seen in case of consolidation, pericardial effusion was seen in $5 \%$, and also $5 \%$ had hyperaeration and in two case mild unilateral pneumothorax was seen.

There are some limitations in this study such as retrospective design and no follow up chest CT scan after recovery of patients. Therefore, it is recommended more research in the future for better evaluation of lung involvement and proper management, and follow up chest CT after completing treatment should be considered. In the study of Fei shan et al. reported that automatically quantify infected lobes and segments and followed up CT scan every 3-5 day during management (12). In a study by Kay et al. 
(33)recommended that focus on the encouraged researchers to evaluate other patterns of lung involvement for accurate diagnosis and atypical features of lung involvement in chest CT.

\section{Conclusion}

CXR is not sensitive method as a first imaging in diagnosis of COVID-19 pneumonia (35\% normal CXR). Chest CT scan is very sensitive but is nonspecific and is not characteristic. Right lower lobe, mostly posterior basal segment is the most common lobe which is involved and the right middle lobe showed minimally and least involvement. Ground glass opacity, predominantly in lower posterior and peripheral distribution, was the most imaging feature which is compatible with non-specific organizing pnumonia and about $12 \%$ showed atypical imaging pattern.

\section{Appendix Files}

1. A COVID 19 positive patient, presenting fever and dry cough for 3 days, a CXR at admission was normal, bilateral GGO in both lower lobes with patchy

2. A COVID 19 positive patient, presenting with fever and dyspnea from 3 days ago. CT scan shows ground glass and crazy paving appearance in left lower lobe and focal reticular density in the right lower lobe and right anterior pneumothorax

3. A COVID 19 positive patient, with sever cough and dyspnea for 5 days' chest $\mathrm{x}$ ray shows sever generalized ground glass opacity and consolidation in both lung (white lung)

4. A COVID 19 positive patient, with presenting with dry cough and dyspnea from 3 days ago CT scan shows typical patchy peripheral and posterior predominant opacity scattered in the both lung

\section{Abbreviations}

COVID-19

Coronavirus Disease 2019

SARS

Severe Acute Respiratory Syndrome

MERS

Middle East Respiratory Syndrome

RT-PCR

Real Time- Polymerase Chain Reaction

SD

Standard Deviation

GGO

Ground Glass Opacity

\section{Declarations}




\section{Ethic approval and consent to participate:}

The protocol was approved by the Committee for Human Research at the Hamadan University of Medical Sciences, Iran. written informed consent for the survey was obtained from all the patients.

\section{Consent to publish:}

Not applicable.

\section{Availability of data and materials:}

It should be noted that these patients have not been reported in any other submission by us or anyone else. All supporting data is available through the corresponding author.

\section{Competing Interest}

The author claimed no conflict of interest.

\section{Funding}

Hamadan University of Medical Sciences financially supported this study. This funding source had no role in analysis and interpretation of data; in the writing of the manuscript; nor in the decision to submit it for publication.

\section{Author's contribution}

FG, SHH, FK and SK developed the original idea and the protocol, abstracted and prepared the manuscript. SK, MG, FG and SB participate in the study design and analyzed the data. SB, FEA, FG, MM, FK and MMM contributed to study design and data gathering. All authors drafted the work. All authors read and approved the final manuscript.

\section{Acknowledgements}

The authors would like to appreciate the Vice-chancellor of Research and Technology, Hamadan University of Medical Sciences for funding the study. Also we would like to thank the Health staffs of public hospitals of Hamadan Province for their kind collaboration.

\section{Authors' Information}

Farideh Gharekhanloo ${ }^{1}$, Seyyed Hamid Hashemi ${ }^{2}$, Fariba Keramat ${ }^{2,3}$, Farzaneh Esna-Ashari ${ }^{4}$, Mojgan Mamani $^{2,3}$, Mohammad Mahdi Majzoubi ${ }^{2}$, Peyman Eini ${ }^{2}$, Mahsa Gharekhanloo ${ }^{5}$, Salman Khazaei ${ }^{6}$

1.Department of Radiology, School of Medicine, Besat Hospital, Hamadan University of Medical Sciences, Hamadan, Iran 
2.Department of Infectious diseases, Faculty of Medicine, Hamadan University of Medical Sciences, Hamadan, Iran

3.Brucellosis Research Center, Hamadan University of Medical Sciences, Hamadan, Iran

4.Department of Community Medicine, Hamadan University of Medical Sciences, Hamadan, Iran

5.Faculty of Art and Architecture, Yazd University, Yazd, Iran

6.Research Center for Health Sciences, Hamadan University of Medical Sciences, Hamadan, Iran

Corresponding author: Salman Khazaei (Ph.D)

Address: Research Center for Health Sciences, Hamadan University of Medical Sciences, Hamadan, Iran

Email: salman.khazaei61@gmail.com

Tell: +98-813-383-0548

\section{References}

1. Zhu N, Zhang D, Wang W, Li X, Yang B, Song J, et al. A novel coronavirus from patients with pneumonia in China, 2019. New England Journal of Medicine. 2020.

2. Bernheim A, Mei $X$, Huang $M$, Yang $Y$, Fayad ZA, Zhang N, et al. Chest $C T$ findings in coronavirus disease-19 (COVID-19): relationship to duration of infection. Radiology. 2020:200463.

3. Hung LS. The SARS epidemic in Hong Kong: what lessons have we learned? J R Soc Med. 2003;96(8):374-8.

4. Li Q, Guan X, Wu P, Wang X, Zhou L, Tong Y, et al. Early transmission dynamics in Wuhan, China, of novel coronavirus-infected pneumonia. New England Journal of Medicine. 2020.

5. Chen N, Zhou M, Dong X, Qu J, Gong F, Han Y, et al. Epidemiological and clinical characteristics of 99 cases of 2019 novel coronavirus pneumonia in Wuhan, China: a descriptive study. The Lancet. 2020;395(10223):507-13.

6. Hui DS, Azhar I, Madani E, Ntoumi TA, Kock F, Dar R. O, et al. The continuing 2019-nCoV epidemic threat of novel coronaviruses to global health-The latest 2019 novel coronavirus outbreak in Wuhan, China. International Journal of Infectious Diseases. 2020;91:264-6.

7. Chan JF-W, Yuan S, Kok K-H, To KK-W, Chu H, Yang J, et al. A familial cluster of pneumonia associated with the 2019 novel coronavirus indicating person-to-person transmission: a study of a family cluster. The Lancet. 2020;395(10223):514-23.

8. Guan W-j, Ni Z-y, Hu Y, Liang W-h, Ou C-q, He J-x, et al. Clinical characteristics of 2019 novel coronavirus infection in China. MedRxiv. 2020.

9. Organization WH. Coronavirus disease 2019 (COVID-19): situation report, 46. 2020. 
10. Wang D, Hu B, Hu C, Zhu F, Liu X, Zhang J, et al. Clinical characteristics of 138 hospitalized patients with 2019 novel coronavirus-infected pneumonia in Wuhan, China. Jama. 2020.

11. Huang C, Wang Y, Li X, Ren L, Zhao J, Hu Y, et al. Clinical features of patients infected with 2019 novel coronavirus in Wuhan, China. The Lancet. 2020;395(10223):497-506.

12. Shan + F Gao + Y, Wang J, Shi W, Shi N, Han M, et al. Lung Infection Quantification of COVID-19 in CT Images with Deep Learning. arXiv preprint arXiv:200304655. 2020.

13. Chung M, Bernheim A, Mei X, Zhang N, Huang M, Zeng X, et al. CT imaging features of 2019 novel coronavirus (2019-nCoV). Radiology. 2020:200230.

14. Hansell DM, Bankier AA, MacMahon H, McLoud TC, Muller NL, Remy J. Fleischner Society: glossary of terms for thoracic imaging. Radiology. 2008;246(3):697-722.

15. Wang Q, Zhang Z, Shi Y, Jiang Y. Emerging. H7N9 influenza A (novel reassortant avian-origin) pneumonia: radiologic findings. Radiology. 2013;268(3):882-9.

16. Li H, Weng H, Lan C, Zhang H, Wang X, Pan J, et al. Comparison of patients with avian influenza A (H7N9) and influenza A (H1N1) complicated by acute respiratory distress syndrome. Medicine. 2018;97(12).

17. Shan F, Gao Y, Wang J, Shi W, Shi N, Han M, et al. Lung Infection Quantification of COVID-19 in CT Images with Deep Learning. arXiv preprint arXiv:200304655. 2020.

18. Xie X, Zhong Z, Zhao W, Zheng C, Wang F, Liu J. Chest CT for typical 2019-nCoV pneumonia: relationship to negative RT-PCR testing. Radiology. 2020:200343.

19. Huang P, Liu T, Huang L, Liu H, Lei M, Xu W, et al. Use of chest CT in combination with negative RTPCR assay for the 2019 novel coronavirus but high clinical suspicion. Radiology. 2020;295(1):22-3.

20. Fang Y, Zhang H, Xie J, Lin M, Ying L, Pang P, et al. Sensitivity of chest CT for COVID-19: comparison to RT-PCR. Radiology. 2020:200432.

21. Yoon SH, Lee KH, Kim JY, Lee YK, Ko H, Kim KH, et al. Chest Radiographic and CT Findings of the 2019 Novel Coronavirus Disease (COVID-19): Analysis of Nine Patients Treated in Korea. Korean Journal of Radiology. 2020;21(4):494-500.

22. Ye Z, Zhang Y, Wang Y, Huang Z, Song B. Chest CT manifestations of new coronavirus disease 2019 (COVID-19): a pictorial review. European Radiology. 2020:1-9.

23. Salehi S, Abedi A, Balakrishnan S, Gholamrezanezhad A. Coronavirus Disease 2019 (COVID-19): A Systematic Review of Imaging Findings in 919 Patients. American Journal of Roentgenology. 2020:1-7.

24. Wu J, Wu X, Zeng W, Guo D, Fang Z, Chen L, et al. Chest CT findings in patients with corona virus disease 2019 and its relationship with clinical features. Invest Radiol https://doi org/101097/RLI. 2020;670.

25. Lee KS, Kullnig P, Hartman TE, Müller N. Cryptogenic organizing pneumonia: CT findings in 43 patients. AJR American journal of roentgenology. 1994;162(3):543-6.

26. Webb WR, Muller NL, Naidich DP. High-resolution CT of the lung: Lippincott Williams \& Wilkins; 2014. 
27. Yuan Y, Tao XF, Shi YX, Liu SY, Chen JQ. Initial HRCT findings of novel influenza A (H1N1) infection. Influenza and other respiratory viruses. 2012;6(6):e114-e9.

28. Wong K-t, Antonio GE, Hui DS, Ho C, Chan P-n, Ng W-h, et al. Severe acute respiratory syndrome: thinsection computed tomography features, temporal changes, and clinicoradiologic correlation during the convalescent period. J Comput Assist Tomogr. 2004;28(6):790-5.

29. Li Y, Xia L. Coronavirus Disease 2019 (COVID-19): Role of chest CT in diagnosis and management. American Journal of Roentgenology. 2020:1-7.

30. Koo HJ, Lim S, Choe J, Choi S-H, Sung H, Do K-H. Radiographic and CT features of viral pneumonia. Radiographics. 2018;38(3):719-39.

31. Shi H, Han X, Jiang N, Cao Y, Alwalid O, Gu J, et al. Radiological findings from 81 patients with COVID-19 pneumonia in Wuhan, China: a descriptive study. The Lancet Infectious Diseases. 2020.

32. Song F, Shi N, Shan F, Zhang Z, Shen J, Lu H, et al. Emerging 2019 Novel Coronavirus (2019-nCoV) Pneumonia. Radiology. 2020:200274.

33. Kay F, Abbara S. The many faces of COVID-19: spectrum of imaging manifestations. Radiological Society of North America; 2020.

\section{Figures}




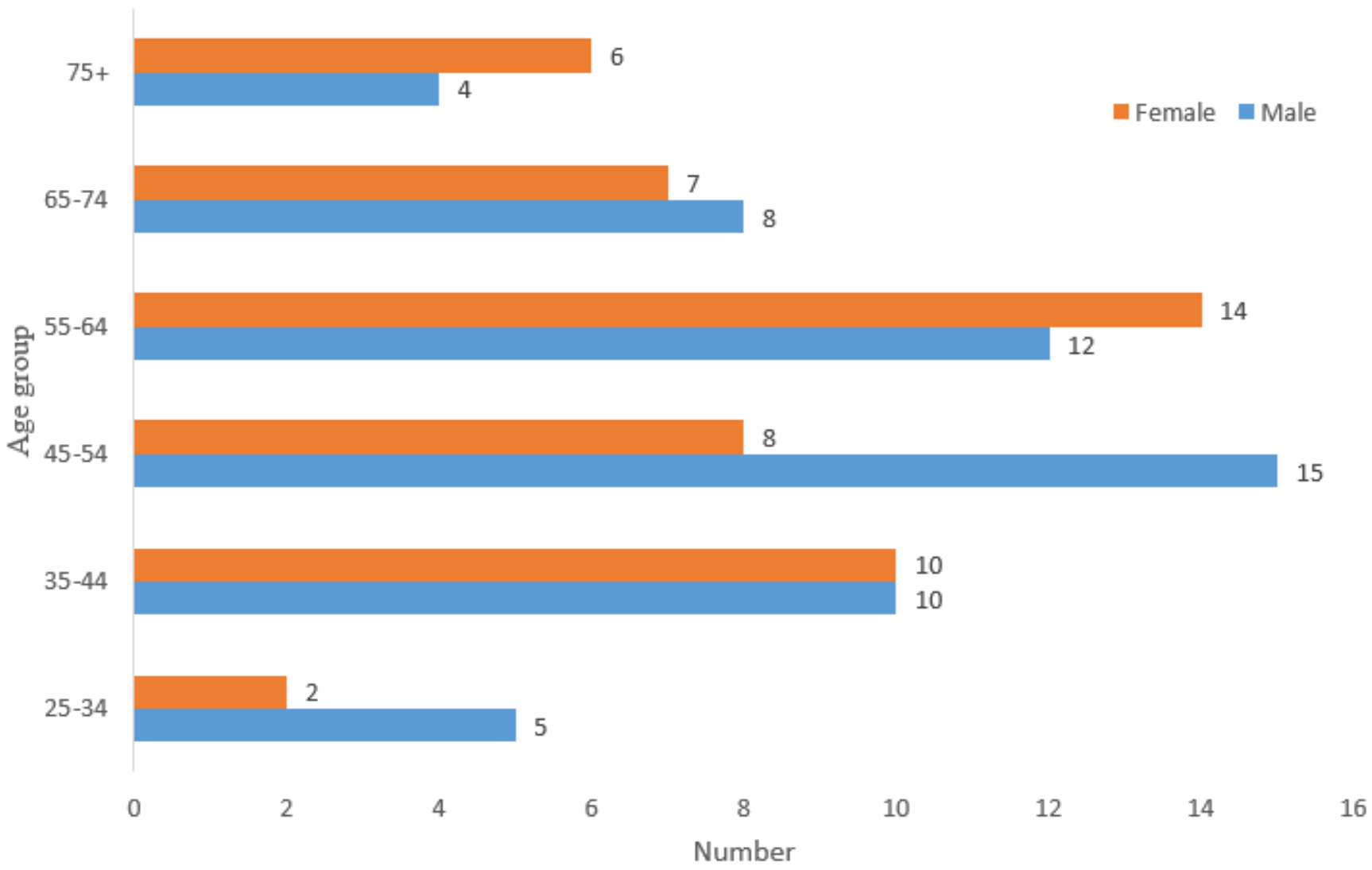

Figure 1

Age distribution of COVID-19 patients by gender 


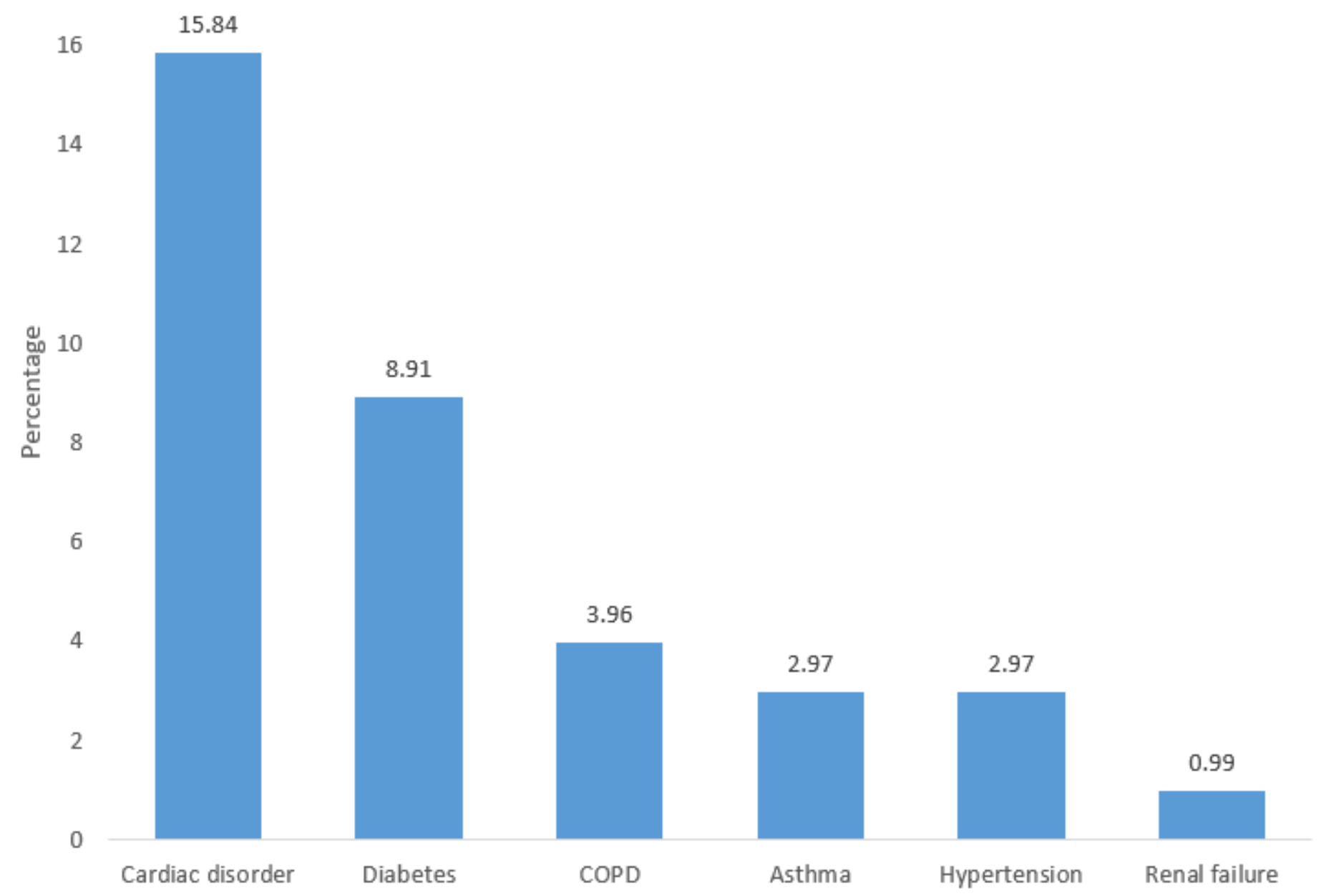

Figure 2

Frequency of underlying diseases in the COVID-19 patients 


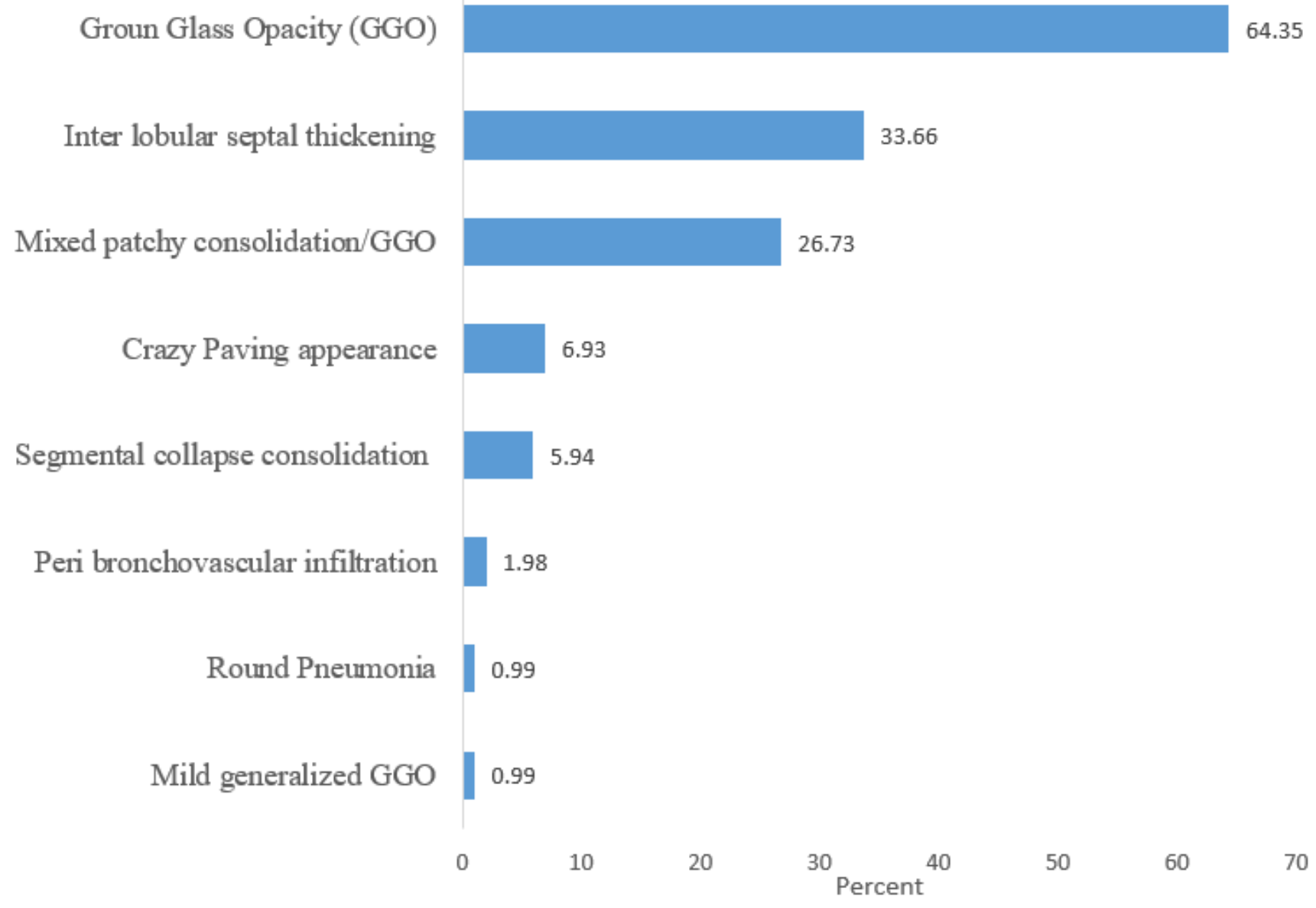

\section{Figure 3}

Chest CT patterns of lung involvement in the COVID-19 patients

\section{Supplementary Files}

This is a list of supplementary files associated with this preprint. Click to download.

- cy.mp4

- hy.mp4

- hh.mp4

- pc.mp4 